DOI: $10.217672573-5365.100017$

\title{
Chemogenomic Profiling: Past, Present and Beyond
}

\author{
Verena Pries and Dominic Hoepfner* \\ Novartis Institutes for BioMedical Research, Novartis Pharma AG, Forum 1 Novartis Campus, CH-4056 Basel, Switzerland
}

${ }^{*}$ Corresponding author: Dominic Hoepfner, Novartis Institutes for BioMedical Research, Novartis Pharma AG, Forum 1 Novartis Campus, CH-4056 Basel, Switzerland, E-mail: dominic.hoepfner@novartis.com

Rec date: Jun 02, 2016; Acc date: June 21, 2016; Pub date: June 23, 2016

Copyright: @ 2016 Pries V, et al. This is an open-access article distributed under the terms of the Creative Commons Attribution License, which permits unrestricted use, distribution, and reproduction in any medium, provided the original author and source are credited.

Citation: Pries V, Hoepfner D. Chemogenomic Profiling: Past, Present and Beyond. Cell Mol Med. 2016, 2:2.

\section{Short Communication}

Drug development of promising hits from phenotypic screens is often hampered due to a lack of information on the cellular target or the mode of action of the compound. An efficient method for target identification is chemogenomic profiling in the surrogate model Saccharomyces cerevisiae. Here we briefly review the progress of this technology, give some successful target identification examples and present future directions for haploinsufficiency and homozygous profiling in higher eukaryotes based on the CRISPR-Cas9 system.

Knowledge of the target protein of a compound is not $a$ priori essential for drug development. However, understanding the mode of action facilitates the informed improvement of a lead structure towards increased potency, higher selectivity, and fewer off-target effects. Target identification by affinitybased methods can fulfil this task but requires a significant chemistry effort. This derivatization is required to define the structure-activity relationship of the hit compound, enabling the binding of the compound to a matrix without interfering with compound-target interactions. Furthermore, affinity proteomics is biased against membrane or low abundance proteins. Haploinsufficiency profiling (HIP) and homozygous profiling (HOP) in the model organism $S$. cerevisiae represent a powerful alternative genetic approach (Figure 1a). It does not require chemical modification of the lead compound and in principle the assay covers all nuclear encoded proteins. HIP exploits the increased sensitivity towards a compound after lowering the dosage of the target-encoding gene from two copies to one copy in diploid yeast [1,2]

The complementary approach to HIP is the homozygous deletion profiling (HOP) where both copies of non-essential genes are deleted. In general, in HOP direct target genes cannot be identified by hypersensitivity but information about functionally related or connected genes that buffer the drugtarget pathway can be gathered (in exceptional cases however if the compound exerts a dominant negative effect on a non- essential gene product it can score as resistant). The feasibility of this approach for identifying molecular targets was demonstrated in pioneering experiments where individual heterozygous strains were grown in the presence of sublethal concentrations of compounds that directly target the gene product of the heterozygous locus [3].

Based on this general protocol we implemented an optimized HIP HOP platform using a fully automated robotic system, testing each substance in duplicate and analyzing negative and positive controls in each assay. Moreover, using the controls for intra-experimental normalization and calculation of additional statistical parameters, we improved the signal-to-noise of the profiles [4]. Applying this screening protocol led to the successful identification of the cellular targets of a number of different compound classes. The feasibility of the approach for target identification of antifungal compounds is demonstrated by the identification of geranylgeranyltransferase I (GGTase I) [5], acetolactate synthase [6] and Erg11p [7].

For these examples, chemogenomic profiling enabled specific follow up studies that highlighted drawbacks of the lead compounds, pinpointing the importance of target knowledge for further drug development. In the case of GGTase I inhibitors it became clear that although the prenylation pathway is conserved among different fungal species it is nonessential in pathogenic species thus challenging the therapeutic value of these scaffolds [5].

Acetolactate synthase was identified as a molecular target of compounds with broad-spectrum antifungal activities. However it became clear that targeting the first step in branched-chain amino acid biosynthesis might be compromised due to the possibility of nutrient bypass in vivo [6]. Identification of Erg11p as antifungal target revealed that the compounds were also active against human cytochrome $\mathrm{P} 450$ s suggesting that substantial medicinal-chemistry efforts might be required to exploit clinical and commercial potential of the compounds [7]. 

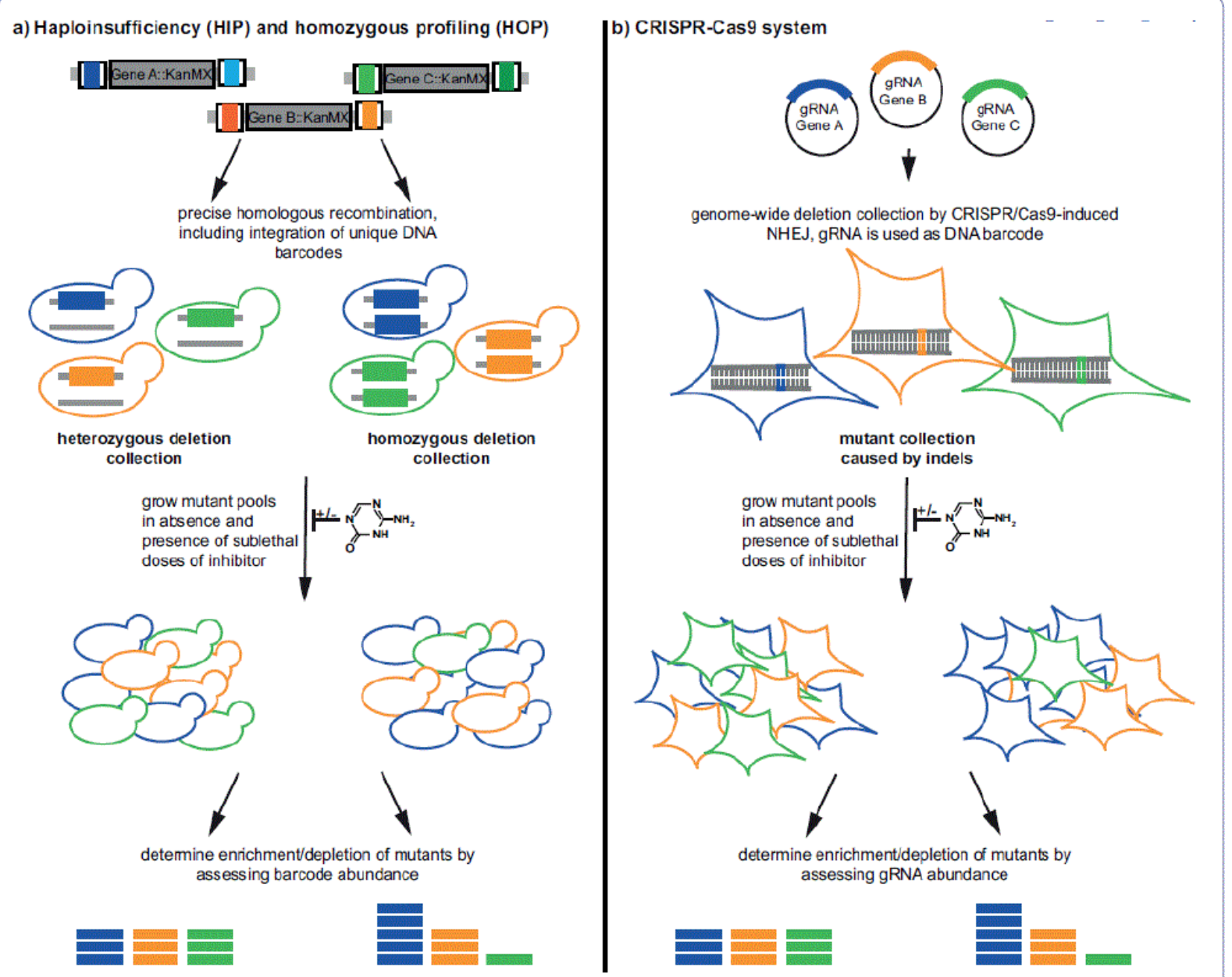

Figure 1: Principle of chemogenomic profiling technologies. Chemogenomic profiling: assessing the relative sensitivity or resistance of different mutant cells in a pool (as measured by over- or under-representation of individual mutants) against sublethal doses of an inhibitor can support identification of affected targets, pathways and resistance factors. a) Genome-wide homozygous and heterozygous deletion collections have been designed for different fungal species taking advantage of efficient homologous recombination in yeasts. When the deletions were generated mutant-specific DNA bar-code sequences were integrated allowing quantification of mutants in complex pools. b) Genome-wide CRISPR-Cas9 gene-editing libraries enable similar experiments in mammalian cells. Double strand breaks induced by the CRISPR-Cas9 system are repaired by error prone non-homologous end joining (NHEJ) resulting in mutations that frequently lead to homo- or heterozygous loss of function and hence resemble a combined HIP-HOP mutant pool of the fungal systems. For quantification of individual mutants the gene-specific 20 nucleotide gRNA sequence is used.

HIP HOP profiling has shown to be useful beyond the discovery of antifungals. Due to evolutionary conservation of many genes and processes HIP HOP can provide target hypotheses for compounds active in other species. For example it supported cytochrome b as the target for GNF7686, an inhibitor of Trypanosoma cruzi [8]. Although cytochrome b is not included in the yeast HIP pool as it is encoded by the mitochondrial genome, the profile indicated that GNF7686 directly interferes with the function of the $S$. cerevisiae respiratory chain at the level of complex III. For cladosporin, a potent natural product inhibitor of Plasmodium falciparum blood- and liver-stage proliferation, chemogenomic profiling highlighted lysyl-tRNA synthetase as cellular target and facilitated target validation in P. falciparum [9]. As highlighted by cladosporin, HIP HOP has proven to be especially efficient when profiling natural products for a number of reasons: first, because natural products are often targeting evolutionary conserved proteins. Second, because HIP HOP is a linker-free technology, and many natural products are not easily amenable to chemical modification, it is ideally suited to studying their mechanism of action.

The cyclic depsipeptide decatransin was found to inhibit growth of HCT116 cells and chemogenomic profiling highlighted the Sec61-Sec63 core complex, machinery for protein translocation and membrane insertion at the ER, as the site of intervention [10]. Rocaglamides are plant-derived natural products with reported insecticidal, antifungal and anticancer activities. Haploinsufficiency profiling of rocaglamide identified numerous sensitive strains 
heterozygous for different components of the translation initiation pathway, suggesting the possible target process, but making target de-convolution difficult. Further experimentation demonstrated that rocaglamides exert a dominant negative effect on translation initiation and the molecular target had been identified as a resistant hit in the profile [11]. Nannocystin A, a cyclic lactone inhibitor isolated from myxobacteria, showed differential activity across various cancer cell lines. Using the HIP HOP platform, $\alpha$-(TEF2) and $\beta$ subunit (EFB1) of the translation elongation factor 1 (EF-1) complex were identified as putative targets [12].

In the case of the natural product novolactone HIP suggested cytosolic and ER-localized isoforms of Hsp70 as the cellular targets, as the yeast ScHsp70, an Hsp70 NEF (SSE1), a ScHsp90 co-chaperone (CNS1) and stress induced heat shock factor (HSF1) were found to be inhibited. In addition the profile was distinct from that of known Hsp90 inhibitors [13]. FR171456 has cholesterol-lowering properties in animal models as well as broad antifungal activity, and HIP revealed the sterol pathway enzyme Erg26p (sterol-4-alphacarboxylate-3-dehydrogenase) as the likely molecular target. This finding supported results from a metabolite-profiling assay where NSDHL (NAD $(P)$ dependent steroid dehydrogenase-like) the human homologue was identified [14].

In the presented examples the compound target identified in yeast was also the conserved compound target in human cells. Indeed, bioinformatics analysis supported that $31 \%$ of the proteins encoded by yeast genes have human homologues and that approximately $50 \%$ of the human genes implicated in heritable diseases are also found in yeast $[15,16]$. Thus there is a good chance that targets identified by chemogenomic profiling in yeast can be extrapolated to higher organisms. This makes $S$. cerevisiae a relevant model organism to study mammalian diseases and pathways.

With the invention of the CRISPR-Cas9 system a new era for genome-wide chemical profiling has started (Figure 1b). A first quantitative CRISPR-Cas9 interference screen in yeast performed by Smith and coworkers enabled library design and profiling in S. cerevisiae (although not yet with genome-wide coverage). Their CRISPR-Cas9 interference screen identified chemical-genetic interactions of 18 small molecules and also revealed a new mechanism for the suppression of fluconazole toxicity [17].

A first genome-wide CRISPR-Cas9 based approach was also executed in mammalian cells to identify the cellular target and mechanism of action of the potent broad-spectrum antiviral compound GSK983. By using shRNA and CRISPR-Cas9 screen in parallel, dihydroorotate dehydrogenase (DHODH), an enzyme responsible for de novo pyrimidine biosynthesis was found to be inhibited by GSK983. This blockage of pyrimidine metabolism explained the antiviral potency on the one hand and observed cytotoxicity in fast dividing cells on the other hand [18].

However Deans and coworkers state that genes required for essential processes can only be identified by shRNA-mediated knockdown and not in CRISPR-Cas9 deletion screens (as complete editing would lead to depletion of the cell from the pool). This suggests that CRISPR-Cas9 screens have the potential to take the yeast HOP assay into mammalian systems. The mechanisms for CRISPR interference (CRISPRi) are still being investigated and optimized but it is clear that this has the potential to also bring HIP into mammalian cells and thus allow chemogenomic profiling for mammalian targets that are not conserved in fungi [19].

The ever growing body of literature around CRISPR-Cas9 demonstrates its applicability beyond mammalian cells in biological systems such as plasmodia or plants $[20,21]$. Thus it will be interesting to see how the profound expertise gained in chemogenomic profiling in yeast will help to build and improve genome-wide CRISPR-Cas9 based technologies in other species. In combination with the current upswing of phenotypic screens [22], chemogenomic profiling enabled by CRISPR-Cas9 could boost and accelerate the urgently needed discovery of effective novel anti-infectives, pesticides and human medicines.

\section{Acknowledgment}

We thank Stephen Helliwell for proofreading the manuscript.

\section{References}

1. Giaever G, Shoemaker DD, Jones TW, Liang H, Winzeler EA, et al. (1999) Genomic profiling of drug sensitivities via induced haploinsufficiency. Nat Genet 21: p 278-283.

2. Winzeler EA, Shoemaker DD, Astromoff A, Liang H, Anderson K, et al. (1999) Functional Characterization of the $S$. cerevisiae Genome by Gene Deletion and Parallel Analysis. Science. 285: 901-906.

3. Giaever G, Chu AM, Ni L, Connelly C, Riles L, et al. (2002) Functional profiling of the Saccharomyces cerevisiae genome. Nature 418: 387-391.

4. Hoepfner D, Helliwell SB, Sadlish H, Schuierer S, Filipuzzi I, et al. (2014) High-resolution chemical dissection of a model eukaryote reveals targets, pathways and gene functions. Microbiological Research 169: 107-120.

5. Pries V, Cotesta S, Riedl R, Aust T, Schuierer S, et al. (2016) Advantages and Challenges of Phenotypic Screens: The Identification of Two Novel Antifungal Geranylgeranyltransferase I Inhibitors. J Biomol Screen 21: 306-315.

6. Richie DL, Thompson KV, Studer C, Prindle VC, Aust T, et al. (2013) Identification and Evaluation of Novel Acetolactate Synthase Inhibitors as Antifungal Agents. Antimicrobial Agents and Chemotherapy. 57: 2272-2280.

7. Hoepfner D, Karkare S, Helliwell SB, Pfeifer M, Trunzer M, et al. (2012) An Integrated Approach for Identification and Target Validation of Antifungal Compounds Active against Erg11p. Antimicrobial Agents and Chemotherapy 56: 4233-4240.

8. Khare S, Roach SL, Barnes SW, Hoepfner D, Walker JR, et al. (2015) Utilizing Chemical Genomics to Identify Cytochrome b as a Novel Drug Target for Chagas Disease. PLoS Pathog 11: e1005058. 
9. Hoepfner D, McNamara WC, Lim SC, Studer C, Riedl R, et al. (2012) Selective and Specific Inhibition of the Plasmodium falciparum Lysyl-tRNA Synthetase by the Fungal Secondary Metabolite Cladosporin. Cell Host \& Microbe 11: 654-663.

10. Junne T, Wong J, Studer C, Aust T, Bauer BW, et al. (2015) Decatransin, a new natural product inhibiting protein translocation at the Sec61/SecYEG translocon. Journal of Cell Science. 128: 1217-1229.

11. Sadlish H, Galicia-Vazquez G, Paris CG, Aust T, Bhullar B, et al. (2013) Evidence for a Functionally Relevant Rocaglamide Binding Site on the elF4A-RNA Complex. ACS Chemical Biology 8: 1519-1527.

12. Krastel P, Roggo S, Schirle M, Ross NT, Perruccio F, et Al. (2015) Nannocystin A: an Elongation Factor 1 Inhibitor from Myxobacteria with Differential Anti-Cancer Properties. Angewandte Chemie International Edition 54: 10149-10154.

13. Hassan $A Q$, Kirby $C A$, Zhou W, Schuhmann T, Kityk R, et al. (2015) The novolactone natural product disrupts the allosteric regulation of Hsp70. Chem Biol 22: 87-97.

14. Helliwell SB, Karkare S, Bergdoll M, Rahier A, Leighton-Davis JR, et al. (2015) FR171456 is a specific inhibitor of mammalian NSDHL and yeast Erg26p. Nat Commun 6: 8613.
15. Forsburg SL (2001) The art and design of genetic screens: yeast. Nat Rev Genet 2: 659-668.

16. Hartwell LH (2004) Yeast and cancer. Biosci Rep 24: 523-544.

17. Smith JD, Suresh S, Schlecht U, Wu M, Wagih O, et al. (2016) Quantitative CRISPR interference screens in yeast identify chemical-genetic interactions and new rules for guide RNA design. Genome Biology 17: p 1-16.

18. Deans RM, Morgens DW, Ökesli A, Pillay S (2016) Parallel shRNA and CRISPR-Cas9 screens enable antiviral drug target identification. Nat Chem Biol 12: 361-366.

19. Gilbert LA, Horlbeck MA, Adamson B, Villalta JE, Chen Y, et al. (2014) Genome-Scale CRISPR-Mediated Control of Gene Repression and Activation. Cell 159: 647-661.

20. Wagner JC, Platt RJ, Goldfless SJ, Zhang F, Niles JC, et al. (2014) Efficient CRISPR-Cas9-mediated genome editing in Plasmodium falciparum. Nat Meth 11: 915-918.

21. Kaya H, Mikami M, Endo A, Endo M, Tokli S (2016) Highly specific targeted mutagenesis in plants using Staphylococcus aureus Cas9. Sci Rep 6: 26871.

22. Mullard A (2015) The phenotypic screening pendulum swings. Nat Rev Drug Discov 14: 807-809. 